\title{
1977 Nobel Prize in Physics
}

The Royal Swedish Academy of Sciences has awarded the 1977 prize equally to Dr. Philip W. Anderson, Bell Telephone Laboratories, Professor Sir Nevill F. Mott, Cambridge University and Professor John H. Van Vleck, Harvard University for "their fundamental theoretical contributions concerning the electronic structure of magnetic and disordered systems".

Although the 1977 prizewinners have worked in a wide variety of areas within solid state theory, a common element in their research has been their concern with the electron-electron and electron-lattice interactions in solids, and the influence of these interactions on localized electron states in magnetic and disordered systems. It is characteristic of them all that they work closely with their experimental colleagues, helping to interpret their results and proposing new lines of research. The advances which they have made in our understanding of solids have been of fun-

\section{A. R. Mackintosh, Copenhagen}

\section{John H. Van Vleck}

Van Vleck was born in 1899 and received his Ph.D from Harvard University in 1922. He worked at the University of Minnesota from 1923-28 and at the University of Wisconsin from 1928-34. He then became Professor of Physics at Harvard, where he has remained ever since. He retired in 1969 .

Van Vleck is generally regarded as the founder of the modern theory of magnetism, and has been responsible for many advances in the subject. His book Electric and Magnetic Susceptibilities from 1932 is a classic work which is still extensively used and has stimulated an enormous amount of research. In it he presented the quantum theory of the magnetism of atoms and ions, of which he was the principal architect, and which is used essentially unchanged today. He introduced a new type of temperatureindependent paramagnetism, now called "Van Vleck paramagnetism", which allowed him to explain the magnetic properties of, for example, europium and samarium, which had previously defied analysis.

Van Vleck carried out pioneering work on the influence of conduction electrons on the magnetism of metals. For example, he made important contributions to the theory of the indirect exchange interaction, mediated by the conduction electrons, which is basic for the understanding of rare earth metals, and considered the effect of electron correlation in producing localized magnetic moments. The physical ideas which he propounded were later developed by his former student, P.W. Anderson, and influenced Mott's theory of metal-insulator transitions. Van Vleck also developed much of the microscopic theory of antiferromagnetism.

Perhaps his greatest impact on the theory of magnetism has been through his work on the crystalline electric field which, together with the exchange interaction, determines the great majority of the magnetic properties of solids. Van Vleck introduced the concept into magnetism and has shown how it is responsible for a variety of static and dynamic phenomena. For example, he demonstrated how the crystal field can quench the orbital angular momentum and, together with the spin-orbit coupling, determine the magnetic properties of transition and rare earth ions in solids. He furthermore drew attention to the unusual effect which can occur when the ground-state is a singlet, and therefore non-magnetic. He elucidated the role of the crystal field in giving rise to magnetic anisotropy and to the Jahn-Teller distortion of the lattice about a localized magnetic moment. $\mathrm{He}$ constructed the first successful theory of the interaction between local moments and the lattice, through the phonon modulation of the crystal field. This work is of fundamental importance for the interpretation of paramagnetic resonance and relaxation.

Although Van Vleck remained active until the 1960's when much of his work on rare earth metals was perfor-

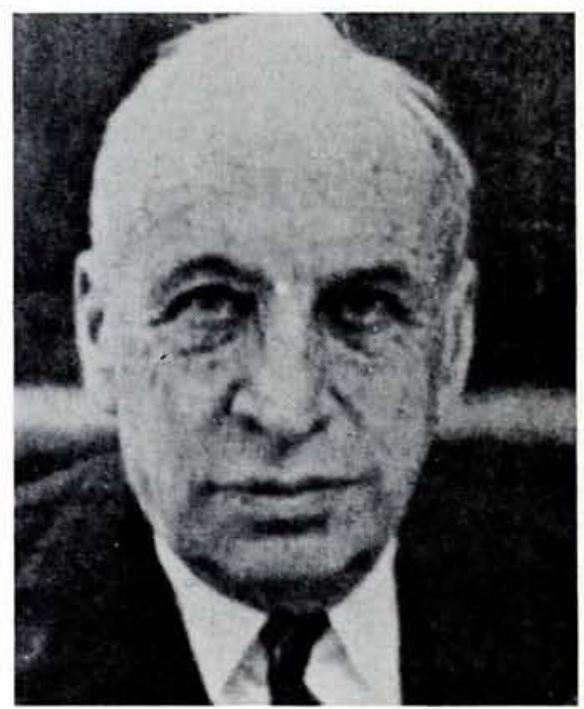

med, many of his most fundamental ideas were proposed decades ago. However, the full significance of many of these ideas has only been appreciated during the flourishing period of experimental studies on magnetic materials in the last decade. In particular, the technique of inelastic neutron scattering has allowed the investigation of many of the phenomena inherent in his work. The intensive examination in recent years of the properties of, for example, singlet ground-state systems, rare earth metals and Jahn-Teller systems has shown the crucial effect of the crystal field on these materials. In such studies, as in so many in the field of magnetism, the interpretation of the results is based upon extensions of Van Vleck's original efforts. 


\section{Nevill F. Mott}

Mott was born in 1905. He was educated at the University of Cambridge following which he became a university lecturer, first at Manchester and then at Cambridge. In 1933 he began his long and fruitful association with the University of Bristol, where he remained as Professor of Physics until appointed Cavendish Professor at Cambrige in 1954. He retired in 1971.

In his long and productive career, Mott has had a crucial influence on the development of solid state physics. His most important achievements lie perhaps in his theories of the physical and mechanical properties of metals and alloys, the metal-insulator transition and, most recently, electronic phenomena in disordered materials. He has contributed decisively to the basic understanding of such important technical matters as fatigue, oxidation, photography and solid state devices.

It has always been characteristic of Mott's mode of working that he discusses his ideas constantly with experimentalists, and provides a fund of suggestions for rewarding new investigations. His way of thinking has especially in later years, been essentially physical rather than mathematical and this has allowed him to communicate with experimentalists on their own terms. He has furthermore been able to take the sometimes abstruse work of solid state theorists and, with his exceptional insight, transform it into accessible form and build a host of new ideas upon it. An excellent example is disordered materials where, in the 1960's, he took Anderson's very important, but at the time little appreciated paper on random lattices from 1958 (discussed further below) and used it as a building block for an essentially new way of thinking about a whole class of solids. His book with E.A. Davis Electronic Processes in Non-Crystalline Materials from 1971 illustrates his qualities of physical intuition and clear exposition.

Perhaps the most remarkable of his achievements was the proposal, first made in 1949 and elaborated in 1956 and 1961, of the existence of the metal-insulator transition which now normally goes under the name "Mott transition". Although it had already been suggested before the war that the insulating properties of $\mathrm{NiO}$ must be due to correlation, and the localized nature of the $4 \mathrm{f}$-electrons in the rare earth metals was implicitly recognized, Mott was the first to place the subject on a sound footing, making predictions which have since been shown experimentally to be generally correct, and stimulating a large amount of fruitful research over the past two decades. The basic applicability of his ideas to ordered systems has been perhaps most clearly demonstrated by experiments on vanadium oxides, while their relevance for the general understanding of solids

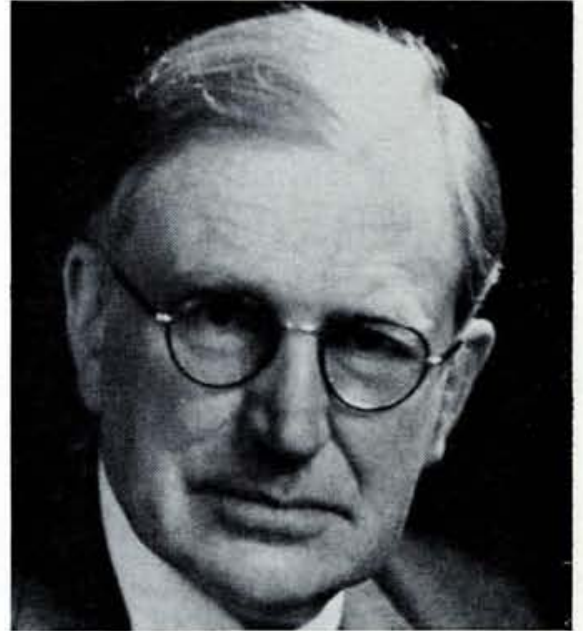

has been strikingly manifested by quite recent work on the properties of electron-hole gases and liquids in intensely illuminated germanium. The whole field is summarized in his recent book Metal-Insulator Transitions of 1974 .

It is perhaps worth emphazing the novelty of Mott's ideas on this subject. Although they are now almost universally accepted, they were initially received with considerable scepticism, because they implied that the band-theory of solids, which had such impressive successes to its credit, is not applicable in some perfect solids under ordinary conditions. In this sense, Mott must indeed be credited with causing a profound and far-reaching change in the way in which we think about solids.

\section{Philip W. Anderson}

Anderson was born in 1923. He received his $\mathrm{Ph}$. D. from Harvard University in 1949 and began working at

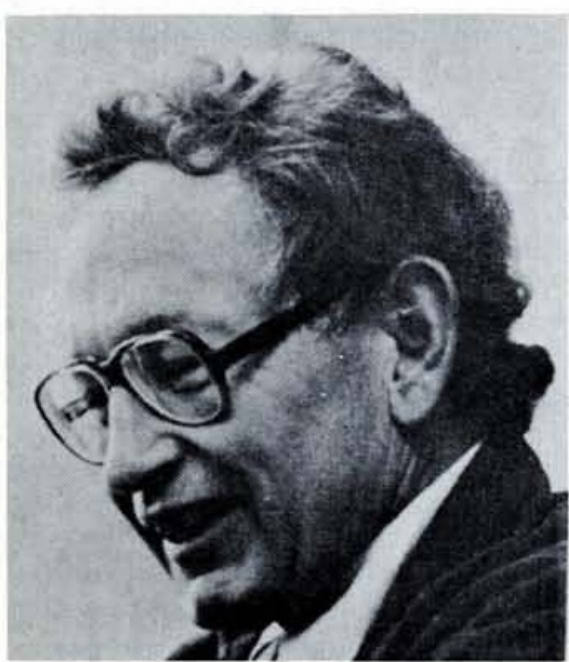

Bell Telephone Laboratories the same year. $\mathrm{He}$ has remained there ever since and now occupies the position of Assistant Director of Physical Research. He was part-time visiting professor at the University of Cambridge from 1967-1975, since when he has held a similar position at Princeton University.

Anderson is an extremely versatile physicist who has made distinguished contributions to a large range of topics; for example, superexchange interactions, impure superconductors, the properties of superfluid ${ }^{3} \mathrm{He}$, disordered materials and localized magnetic moments. His impact on solid state physics has been very great, both through the major influence which he has had on the development of the outstanding research programme at Bell Laboratories, and through the effect of his penetrating and original ideas.

As has been mentioned above, Anderson's paper "Absence of Diffusion in Certain Random Lattices" published in 1958 provided the basis upon which most of the modern theory of electronic states in disordered systems rests. In it he demonstrated that randomness in the potential can itself be sufficient to cause localization of the electron states, and hence the absence of conductivity. The quantitative calculation of this effect is very difficult, and Anderson's original estimates have been improved, but the basic idea is accepted as correct and of fundamental importance. Mott coined the phrase "Anderson localization" to describe this phenomenon.

In his paper "Localized Magnetic States in Metals" from 1961, Anderson 
gave a description of the effect of the Coulomb correlation on producing localized magnetic states in metals. This paper, which is extremely elegant and clear, gave a great impetus to the study of dilute magnetic alloys, a field which has proved to be full of interesting phenomena and which continues to be of active interest today. Anderson has continued to work in this field and has suggested new approaches to the Kondo problem of the interaction between an isolated moment and the conduction electrons, and to that of disordered systems of localized moments, the so-called "spin-glass" problem, again giving stimulus and direction to the efforts of many experimentalists. The latter problem provides a nice analogy with his work on disordered non-magnetic systems. He has made a major contribution to the great development which has occured in the last two decades in our understanding of the behaviour of localized moments in metals and of disordered systems.

Both Van Vleck and Mott were pioneers in establishing the field of solid state physics and have made vital contributions to the subject. Anderson is from a younger generation, but his work is also widely recognized as being of the greatest importance for our understanding of the solid state. The award of the Nobel prize to these three very distinguished scientists will give rise to great satisfaction and pleasure throughout the physics community.

\section{EPS CONFERENCE REPORTS}

\section{Workshops of the Solar Physics Section}

A main objective of the Solar Physics Section is to formulate suitable topics for workshops and to arrange for such workshops to be organized. As the idea of a workshop is to bring together a number of specialists to work jointly on problems of common interest, these may come closer to a solution during the workshop itself, and agreements on continued coordinated effort may be reached. A report on the workshop on Pluridimensional Radiative Transfer held in September 1976 appeared in Europhysics News, 8 (1977) 4 and short accounts are now given of the three workshops held in 1977. For 1978 , two workshops are planned: a workshop on Type I Solar Radio Emission (the so-called noise storms), to be held in March in Toulouse and a workshop on the Sun's Granulation, to be held in the Autumm in Freiburg.

\section{Atomic Data and the Solar Maximum} Mission, Zürich, April 1, 1977.

This meeting, organized by $M$. Huber and $\mathrm{H}$. Nussbaumer, was attended by 33 scientists from Belgium, England, France, Germany, Holland, Italy and Switzerland. The capabilities of the Solar Maximum Mission (SMM) instruments were presented together with a review of the present state of solar physics of relevance to EUV and X-Ray observations. Representatives of individual groups reported about their activities and plans relating to SMM. The information thus exchanged was very valuable to the participants. However, it became obvious that a one day meeting was not of sufficient length to treat problems in any depth but the meeting acted as a first com- prehensive European information exchange concerning SMM ; in this respect it fulfilled a very useful purpose which was appreciated by all participants.

Measurements and Interpretation of Polarization arising in the Solar Cromosphere and Corona, Lund, May 9-13.

This meeting dealt with polarization which arises primarily from coherent scattering in spectral lines and from the Zeeman effect in the presence of magnetic fields. The conditions in the chromosphere and the corona are fundamentally different from the lowerlying photosphere, as the radiation and matter are far from a state of thermodynamic equilibrium due to the low density and small collision rate. Particular attention was paid to a new research area, the study of coherence effects in radiative scattering in a magnetic field (the Hanle effect), caused by interference between the wavefunctions of the partially overlapping Zeeman sublevels of the excited level. These coherence phenomena may provide a future tool for determining vector magnetic fields in the chromosphere and corona.

Twenty five scientists from 10 countries participated in the workshop. The organizing committee consisted of J.O. Stenflo (chairman), D. Dravins and L.G. Stenholm. The proceedings have been published in a preprint volume which can be obtained free of charge from the Librarian, Lund Observatory, Svanegatan 9, S-222 24 Lund, Sweden. Its title is :

Proceedings of a Workshop on Measurements and Interpretation of Polarization Arising in the Solar Chromo- sphere and Corona, Ed. J.O. Stenflo, Reports from the Observatory of Lund No. 12, 1977.

Heliography on Coronal Active Regions, Düsseldorf, June 18-23..

This meeting, organized by $\mathrm{O}$. $\mathrm{Ha}-$ chenberg, W. Hirth and E. Fürst, was attended by 24 scientists.

Three topics, introduced by lectures, were discussed:

a) X-ray pictures of the sun, by $G$. Elwert;

b) Radio emission from active regions, by M. Pick ;

c) Theoretical aspects of hot active regions, by L.E. Cram.

Keen attention has been paid to the bright cores in active regions the "temperature" being a few million degrees up to $10^{7} \mathrm{~K}$ when observed at short $\mathrm{cm}$-radio waves. At present, the accuracy of the measurements is not sufficient to decide whether these core temperatures agree with the EUV-ones, derived from satellite observations. There is a slight indication that they are in disagreement, raising the possibility of there being a non-thermal origin of the radio radiation. This has resulted in a proposal for combined EUV- and radio observations in the future.

Besides this problem, the development of the bright cores into flarelike features was considered. This problem is related to the development of the magnetic field structure.

A summary of the workshop is being prepared and will be available towards the end of 1977.

A. D. Fokker 